
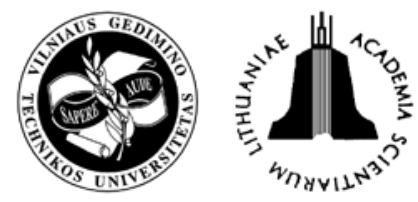

TRANSPORT

2008

23(4): 291-298

\title{
METRICS FOR PERFORMANCE EVALUATION OF SAFETY APPLICATIONS IN VEHICULAR AD HOC NETWORKS
}

\author{
Saleh Yousefi ${ }^{1}$, Mahmood Fathy ${ }^{2}$ \\ Computer Engineering Faculty, Iran University of Science and Technology, Narmak \\ 16846-13114 Tehran, Iran \\ E-mails: ${ }^{1}$ syousefi@iust.ac.ir; ${ }^{2}$ mahfathy@iust.ac.ir
}

Received 22 April 2008; accepted 3 October 2008

\begin{abstract}
In the recent years, direct message exchange between vehicles in order to improve the safety of road traffic has been attracting lots of interest in both networking and road safety communities. While travelling on a road, vehicles form an ad hoc network called Vehicular Ad hoc NETwork (VANET) and deploy life safety applications. Evaluating the performance of these applications is primordial for realizing VANETs in real life. Current literature lacks efficient ways to evaluate the performance of safety applications and mostly leverages on classical networking metrics like delay, delivery rate etc. In this paper, we consider both networking and safety concerns simultaneously to come up with more efficient methods. In particular, we first point out the significance of fairness and coverage from safety viewpoint. Then, we introduce two new metrics called beaconing rate and effective range aiming at providing more facilities for safety performance evaluation in VANET's research. Furthermore, realizing special characteristics of safety applications while disseminating beacon messages, we study the way that beacon dissemination protocols affect the performance of safety applications. We then conduct extensive simulation study to show the usefulness of the introduced metrics and derive some insights on the feasibility of driver-assistant safety applications. Our evaluation also shows that sending the aggregated status of neighbouring vehicles in addition to vehicle's own status, and instead, increasing beacon transmission interval may be invoked in order to assist safety applications in providing satisfactory services to drivers.
\end{abstract}

Keywords: VANETs, safety application, metric, effective range, beacon safety messages, beaconing rate.

\section{Introduction}

Each year, many people suffer from different traffic causalities around the world. This issue followed by the huge economic burden of accidents urges governments to improve the level of safety on roads. In 70's, passive safety systems like safety belts and airbags were introduced. Although they have decreased the severity of accidents and the number of kills and injuries, passive safety systems have not been able to decrease the number of accidents, and therefore active safety systems like the ABS (Antilock Brake System) and ESP (Electronic Stability Program) systems have been invented. The DOT HS 809 767 (2003) statistics shows that the active safety systems have stopped a rising trend of accidents but the number of accidents remains almost the same for many years. In other words, it seems that the current safety systems are not able to decrease the number of accidents any more.

To tackle the aforementioned problem, a lot of investigations have been performed in order to understand the effective factors causing accidents. The results of studies in many countries e. g. (Adler 2006), show that information error has the highest impact on accidents. By the information error, we mean that the driver receives critical information either too late or the process is failed. The key factor here is the response time of the driver which is relatively high (more than $1.0 \mathrm{~s}$ by Roess et al. 2004) such that he/she can not react promptly. As a result, if we could somehow increase the information horizon of the driver such that he/she could be informed about distant events earlier, it might lead to a noticeable decrease in the number of accidents as well as the number of kills and injuries.

On the other hand, recent advances in wireless technology make the idea of 'communication anytime and anywhere' more reachable. Inspiring from this idea, there is growing belief that embedding short range wireless radios into vehicles may be quite beneficial in safety aspects. The Federal Communications Commission (FCC) in FCC 03-324 (2004) report has allocated 75 $\mathrm{MHz}$ of spectrum in the $5.9 \mathrm{GHz}$ band for Dedicated Short Range Communication (DSRC) to enhance the 
safety and productivity of the national transportation system (Jarašūnienè, 2007). DSRC ruling has permitted both safety and non-safety (commercial) applications and provided safety messages are assigned priority. As a part of DSRC standard, IEEE Standard 802.11p (2004) improves IEEE 802.11 to deal with vehicular environment which includes data exchange between high-speed vehicles and between the vehicles and the roadside infrastructure. Furthermore, this issue is investigated in many inter-national and national projects like Integrated Project PReVENT (2008) in Europe, InternetITS Consortium Project (2008) in Japan and Networks on Wheels (NOW) Project (2008) in Germany.

Recently, there has been a surge of interest in different aspects of VANETs. A comprehensive survey of the previous works is provided in researches by Yousefi et al. (2006). According to FCC frequency allocation, we can categorize two main classes of applications for vehicular ad hoc networks: comfort and safety.

In comfort applications, the goal is to improve passenger comfort and traffic efficiency. Traffic-information system, route optimization, electronic toll collection, map download, video download and the Internet transactions are the examples of this category. These applications are predicted to grow very fast in the near future.

In safety applications, the goal is to improve the safety level of passengers by exchanging safety relevant information between vehicles. The information is either presented to the driver or used by an automatic active safety system. Cooperative forward collision warning, left/right turn assistant, lane changing warning, stop sign movement assistant and road-condition warning are the examples of such systems. Due to the stringent delay requirements, the applications of this class may demand direct vehicle-to-vehicle communication.

Each safety application requires some message exchange between vehicles. These massages are classified into two categories including alarm and beacon and have different dissemination policies and roles in safety improvement. Alarm messages are issued by vehicles to inform others about the already happened events in a specific point of a road like car crash, icy surface etc., whereas, vehicles issue beacon messages periodically and each vehicle sends its normal status including speed, position, acceleration etc. to the neighbours. Beacon messages are used to inhibit the possible (not already happened) events like erroneous lane changing, forward collisions, wrong left/right turning etc. Note that the messages mentioned above are complementary to each other. While alarm messages may be able to notify the driver in time of already happened events in order to prevent more incidents, beacon messages can prevent from many incidents before they take place. Fig. 1 shows an example of a beacon based safety application, i.e. lane changing assistant. Each vehicle, for instance vehicle $\mathbf{A}$, collects up-to-date status information (e. g. position, speed, acceleration etc.) about its neighbouring vehicles. Then, based on the status of vehicle $\mathbf{B}$ and $\mathbf{C}$, the lane changing assistant application may warn the driver of vehicle $\mathbf{A}$ in case he/she makes a wrong decision to change the lane.

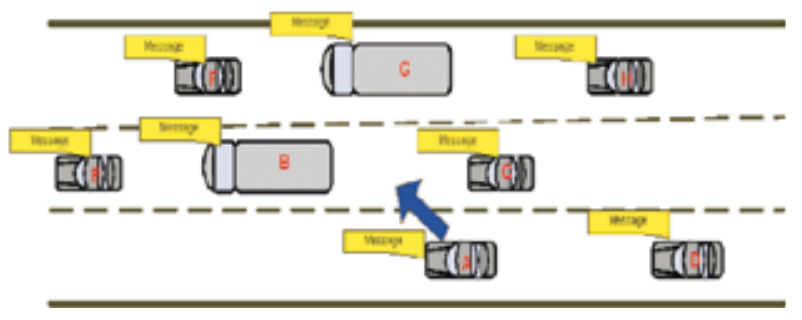

Fig. 1. An example of beacon based safety applications

Safety applications have some special characteristics distinguishing them from ordinary networking applications like Web, FTP etc. Thus, evaluating their performance necessitates simultaneous consideration of both safety and networking concerns. To the best of our knowledge, this issue has been evaded in the current literature. The previous works by Torrent-Moreno et al. (2004, 2005), Xu et al. (2004), Korkmaz et al. (2004) and Yousefi et al. (2007a and 2007b) usually evaluate safety applications in VANETs by means of QoS metrics like delivery rate, delay etc. Although the metrics inherited from networking literature are valuable in many aspects, they are unable to capture distinctive characteristics of safety applications like coverage and fairness (see Chapter 2). In this paper, we intend to fill the gap and introduce two new metrics called effective range and beaconing rate, in particular for evaluating the performance in safety scenarios. The proposed metrics take into account safety as well as networking considerations. We further model the effect of QoS offered by the lower layers of the protocol stack on the performance of safety applications. Based on simulation study and using our metrics, we derive some insights on the dissemination policy of beacon messages as well as the near-term deploying feasibility of driver-assistant safety applications. In the sequel of this paper, we focus on beacon based safety applications, although some discussions can also be applied to the alarm based safety applications.

The rest of the paper is organised as follows. Chapter 2 highlights the distinctiveness of safety scenarios in comparison to common networking scenarios. Chapter 3 looks after the way that beacon dissemination protocols affect the performance of related safety applications. Chapter 4 presents simulation study to show the efficacy of the introduced metrics and derive some interesting insights on the performance of different safety applications as well as on the dissemination policy of beacons. Finally, Chapter 5 concludes the paper.

\section{Evaluation Metrics for Safety Scenarios}

In order to deploy safety applications in VANETs, there should be effective ways to evaluate their degree of success in providing safety. For this purpose, inheriting from networking literature, researchers utilize common evaluation metrics like delivery rate, delay etc. Although these metrics are also valuable for evaluating the performance of safety applications, the following distinctive characteristics necessitates specific attention to the performance evaluation of safety applications: 
- In safety applications, lack of fresh information makes each individual vehicle a life threat for the others. Thus, in order to evaluate the performance of a message dissemination protocol or a safety application, quality of the safety offered to all individual vehicles is critical and should be monitored. While in ordinary networking scenarios usually, the average values of the metrics of interest are usually evaluated, in safety scenarios, the average values are not useful any more. Therefore, we propose to monitor the metrics of interest for all vehicles and count on the worst case values.

- Although the distance between senders and receivers may be important in some networking scenarios, from safety viewpoint, it is the most important metric of performance. To have a solid evaluation in safety scenarios, the coverage property is needed to be evaluated. For any given vehicle the range which the safety applications can cover adequately is of ultimate importance. Within this range, vehicles can receive the beacons of the beacon initiator with satisfactory QoS level, i.e. desirable values for delay, delivery rate etc.

- The definitive goal of any beacon based safety application deployed in a given vehicle is to inform neighbouring vehicles about the vehicle's own status. Hence, evaluating the fact that how well and how fair the beacon dissemination protocols and/ or safety applications are successful in this regard is indispensable from the safety viewpoint.

Note that the above concerns should be taken into account all together. With this in mind, we propose two new metrics, primarily focused on evaluating the performance in safety scenarios as follows:

- Effective range: We define the range within which the worst case of QoS metrics is satisfied as the effective range. The satisfaction levels depend on the projected safety application. Although one may consider a different quality of service metrics, in this paper, we define the effective range as the range within which:

a) the minimum delivery rate is above a predefined threshold;

b) the maximum end-to-end delay is below a pre-defined threshold.

- Beaconing rate: For a given beacon disseminating vehicle, the beaconing rate is defined as the average value between its worst case delivery rates to all surrounding vehicles in its transmission range. This metric assists in knowing how well the beacons disseminating from a vehicle reach other vehicles. When we compare the value of this metric for different vehicles, it gives us valuable insights on the capability of the safety application in providing a fair safety performance to each vehicle.

In the DOT HS 809859 (2005) - Vehicle Safety Communication Project - 34 vehicle safety applications enabled or enhanced by VANETs were studied. Among those, 8 applications were identified as high priority and selected for extracting their communication requirements. These applications include both alarm based and beacon based safety applications. This paper focuses on safety applications deployed based on beacon message disseminations. Typically, safety applications have the following communication requirements:

- communication ranges from $50 \mathrm{~m}$ to $300 \mathrm{~m}$;

- safety message transmission interval from $20 \mathrm{~ms}$ to $1 \mathrm{~s}$;

- safety message size from 200 bytes to 500 bytes;

- delays below $150 \mathrm{~ms}$.

Note that DSRC is based on IEEE 802.11a which currently supports about $300 \mathrm{~m}$ transmission range. Furthermore, it is expected that the communication range reaches 1000 meters when commercial products are available (IEEE Standard 802.11p 2004). Therefore, it could be quite reasonable to consider single-hop dissemination as an important type of future inter-vehicle communications. However, in particular for alarm and comfort messages, multi-hop message propagation as well as multi-hop connectivity is challenging and has been studied in some recent works, for example, Yousefi et al. 2008, Resta et al. 2007. Note that, when we focus on 1-hop beacon dissemination, we get involved in the MAC layer broadcasting which is quite different from network layer broadcasting (beacon messages normally need single-hop broadcasting at MAC layer while alarm safety messages and comfort messages usually demand multi-hop roadcasting/unicasting in the network layer).

\section{Application Layer Performance}

As we know, network protocol stack is structured in a layered architecture in which each layer offers services to its immediate upper layer. The application layer is the up-most layer in the protocol stack and offers services to the user. To ease explanation, we define a virtual layer called Communication layer as the combination of all layers sited under the application layer. This virtual layer normally, as the OSI reference model suggests, includes physical layer, MAC layer, networking layer and transport layer. However, VANETs may be deployed with a rather different protocol stack and some layers may be absent (Füßler et al. 2005). For example, normally in beacon dissemination applications only MAC and physical layer are present. Albeit in the sequel of this section, we continue with the general term 'Communication layer' to deal with more general cases. Fig. 2 shows the architecture we consider in this paper.

The Communication layer offers services (i. e. sending and receiving data) to the application layer. In general, in the networking literature, there are two kinds of relationship between the QoS offered by the Communication layer to the application layer and the one that application layer offers to the users:

- In reliable data transfer protocols like HTTP and FTP, the Communication layer must guarantee reliability in terms of delivery rate even though it may lead to a large delay. Indeed the role of the transport layer is to compensate the shortcom- 


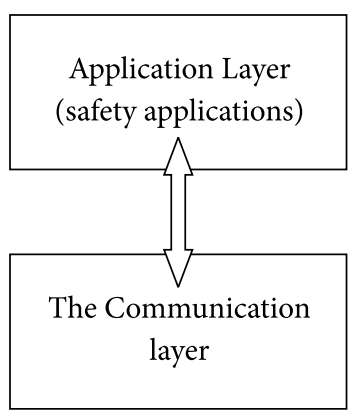

Fig. 2. The two layer architecture

ings of lower layers, so that, the application layer can offer satisfactory services to the user.

- In real time applications, normally it is not required that the Communication layer offers fully reliable services to the application layer because, it may come at the expense of sacrifices of delay which is critical in those applications. In other words, the application layer tolerates some unreliability in the services offered by the Communication layer.

While alarm based safety applications may be put in the first category, we believe that beacon based Safety applications can be categorized in the second group. Note that beacon messages do not contain unpredicted information (as the alarm messages do). Thus, if the application layer does not receive fresh beacon messages from some vehicles for a short period, it can perform extrapolation to guess their status (e. g. speed, position, acceleration etc.), see research by Bai and Krishnan (2006). It should be emphasized that extrapolation is not possible in case of alarm messages because they announce unpredicted events in which the current status of vehicles does not follow their previous status. Furthermore, when a fresh beacon message is received, it actually expires the validity of the previous one. Therefore, it is not needed that the Communication layer tries to re-transmit the old one. These considerations make us convinced that beacon based safety applications can get advantage of some degree of tolerance for QoS offered by the Communication layer. In other words, they function properly even though the Communication layer is not able to offer a desirable quality of service. Recent studies, for example, by Yousefi et al. (2007a) and Yin et al. (2004), on DSRC show that single-hop beacon dissemination in VANETs is adequate in terms of delay but the reliability remains defective. Hence, in the following, we concentrate on reliability as the main performance concern.

For a given vehicle, we define a time window $T$ by which the safety application must receive at least one fresh beacon from any neighbouring vehicles. Denote by $t$ the transmission interval of the Communication protocol. Let $P_{c o m}$ be the success rate of the Communication protocol which is the minimum between all delivery rates in a given transmission range of vehicles. Now, the beacon based safety application works adequately if at least one beacon among $N=T / t$ beacons from each neighbouring vehicle is received. In other words:

$$
\begin{aligned}
P_{a p p}(T, t) & =1-P(\text { all failur in } N \text { tries }) \\
& =1-\left(1-P_{c o m}\right)^{N} \\
& =1-\left(1-P_{\text {com }}\right)^{T / t}
\end{aligned}
$$

where: $P_{a p p}$ is the success rate of the safety application. It should be emphasized that the above equation is valid only if the message losses are independent, as we assume here. Having equation (1), one can simply relate the reliability of the application layer to that of the Communication layer.

In order to design a safety application, the required attribute of the safety application should be extracted by safety experts and then be used by VANET protocol and application designers. So far, in our model each application is attributed by two parameters $T, t$. In addition, we define a vector $\alpha$ representing the satisfaction levels for each quality of service metric (e. g. delivery rate, delay, etc.).

Table 1 shows our proposed attributes for each safety application.

Table 1. Attributes of safety applications

\begin{tabular}{cl}
\hline Attributes & \multicolumn{1}{c}{ Description } \\
\hline$T$ & $\begin{array}{l}\text { The time window of the beacon based safety } \\
\text { application. }\end{array}$ \\
$t$ & $\begin{array}{l}\text { The transmission interval of the beacon } \\
\text { Communication protocol. }\end{array}$ \\
& $\begin{array}{l}\text { A vector containing the satisfaction levels for QoS } \\
\text { metrics of interest (e. g., delay, delivery rate, etc.). }\end{array}$ \\
\hline
\end{tabular}

Based on the above attributes, we can consider different classes of safety applications. In the simulation study done in this paper, we assume the following classes of applications:

- Driver-assistant safety applications are expected to assist the driver in different maneuvers like lane changing, turning left/right etc. This class of applications can prevent from several incidents which may happen because of driver's error. Considering experiments and depending on specific application, $95 \% \leq \alpha \leq \% 99$ and $N \leq 3$ are reasonable values for this class.

- Automatic safety applications are expected to control the vehicle as stand alone systems. Undoubtedly, this class of applications demand very high QoS satisfaction level above $99 \%$ and $N=1$.

\section{Simulation Study}

In this section, we conduct simulation study using GloMoSim 2.03 (GloMoSim Network Simulator 2008), a well known network simulator, and evaluate the performance of some typical safety applications. The goal is, first, to show the effectiveness of our newly introduced metrics and second, to derive some insights on the performance of beacon based safety applications. For this purpose, a single-hop beacon dissemination protocol has been implemented. The road traffic scenario is chosen as follows. We simulate a forced-flow (Roess et al. 2004) traffic situation in which 600 cars are moving in an 8-lane highway (or 4-lane per direction). The density of vehicles is 50 
[veh/ $\mathrm{km} / \mathrm{lane}]$. The vehicles are moving with constant speed $100[\mathrm{~km} / \mathrm{h}]$ or $120[\mathrm{~km} / \mathrm{h}]$. In such a dense network, every vehicle disseminates beacons to inform other vehicles about its status, and thus saturated medium is the main communication challenge. To achieve a better performance, previous works tried to amend the MAC layer (Torrent-Moreno et al. 2004; Xu et al. 2004; Korkmaz et al. 2004 and Yin et al. 2004) or control the power (Torrent-Moreno et al. 2005). While as a complementary approach, we tune the parameters of the application layer (Yousefi et al. 2007a and 2007b). The most effective factors from the application point of view, as DOT HS 809859 (2005) suggests, are as follows: Vehicle's transmission range, Message dissemination transmission interval and Safety message size.

Although there is no standard scenario in the literature, we find it reasonable to use the result of DOT HS 809859 (2005) and implement the following three scenarios:

- Scenario 10P200B: All vehicles send 200 bytes packets every $100 \mathrm{~ms}$. In other words, each vehicle sends 10 packets of 200 bytes data every second.

- Scenario 5P200B: All vehicles send 200 bytes packets every $200 \mathrm{~ms}$. In other words, each vehicle sends 5 packets of 200 bytes data every second.

- Scenario 10P100B: All vehicles send 100 bytes packets every $100 \mathrm{~ms}$. In other words, each vehicle sends 10 packets of 100 bytes data.

Note that the above scenarios are a feasible subset of the scenarios suggested in DOT HS 809859 (2005). We further consider $10 \%$ jitter in transmission intervals. From the networking point of view, the main cause of performance impairment in such a dense network is collisions between neighbours' beacon dissemination. Table 2 shows more details about the simulation parameters.

Table 2. Simulation setting parameters

\begin{tabular}{|c|c|}
\hline Propagation model & Two-ray-ground \\
\hline Transmission range $[\mathrm{m}]$ & $\begin{array}{llllll}50 & 100 & 150 & 200 & 250 & 300\end{array}$ \\
\hline Carrier sense range & $\begin{array}{l}\text { About twice the transmission } \\
\text { range }\end{array}$ \\
\hline MAC type & $\begin{array}{l}\text { IEEE } 802.11 \text { (the base for } \\
\text { DSRC standard) }\end{array}$ \\
\hline Channel bandwidth [Mbps] & 6 \\
\hline Traffic type & CBR (UDP) \\
\hline $\begin{array}{l}\text { Period of message } \\
\text { dissemination }[\mathrm{ms}]\end{array}$ & 100200 \\
\hline Message payload size [byte] & $100 \quad 200$ \\
\hline Number of vehicles & 600 \\
\hline Speed $[\mathrm{km} / \mathrm{h}]$ & 100120 \\
\hline $\begin{array}{l}\text { Traffic density }[\mathrm{veh} / \mathrm{km} / \\
\text { lane] }\end{array}$ & 50 \\
\hline Number of lanes & 8 \\
\hline Simulation time $[\mathrm{sec}]$ & 60 \\
\hline
\end{tabular}

\subsection{The Effective Range of the Beacon Dissemination Protocol}

As a first step, we evaluate the implemented beacon dissemination protocol by means of effective range defined in Chapter 2. Here it may be interesting to give some insights on the performance of the beacon dissemination protocol. Since the MAC layer is based on IEEE 802.11, the performance of the dissemination protocol depends on the amount of load imposed by vehicles to the shared medium. For a given scenario, the channel load is obtained as follows:

$$
\text { channel_load }(\text { bit } / s)=\frac{2 L R}{d} \cdot \frac{1}{T_{I}} \cdot P,
$$

where: $L$ represents the number of lanes, $R(\mathrm{~m})$ represents transmission range, $d(\mathrm{~m})$ represents average inter-vehicle distance, $T_{I}(\mathrm{~s})$ represents message dissemination interval time, and $P$ (bits/packet) stands for message size in bits.

Table 3 shows the channel loads for the above-mentioned scenarios at different transmission ranges.

Table 3. Channel load in the scenarios for different transmission ranges

\begin{tabular}{ccccccc}
\hline Scenario & $50 \mathrm{~m}$ & $100 \mathrm{~m}$ & $150 \mathrm{~m}$ & $200 \mathrm{~m}$ & $250 \mathrm{~m}$ & $300 \mathrm{~m}$ \\
\hline 10P200B & $14 \%$ & $27 \%$ & $40 \%$ & $54 \%$ & $67 \%$ & $80 \%$ \\
5P200B & $7 \%$ & $14 \%$ & $20 \%$ & $27 \%$ & $34 \%$ & $40 \%$ \\
10P100B & $8 \%$ & $16 \%$ & $24 \%$ & $32 \%$ & $40 \%$ & $48 \%$ \\
\hline
\end{tabular}

Fig. 3 shows the effective range of the beacon dissemination protocol versus vehicles' transmission range. To obtain the effective range, we measure the range at which the minimum delivery rate is higher than a threshold, i. e. $\alpha=90 \%$, as well as the maximum delay is below $150 \mathrm{~ms}$. As it is followed from the figure, the scenario 10P200B offers very low effective range such that it can not be considered as a feasible scenario. Moreover, in the scenario $10 \mathrm{P} 200 \mathrm{~B}$, for transmission range of $300 \mathrm{~m}$, the effective range is taken zero because the maximum achievable delay is above $150 \mathrm{~ms}$. Among the other two scenarios, 5P200B offers noticeable higher effective ranges. Table 3 shows that the imposed load of both scenarios

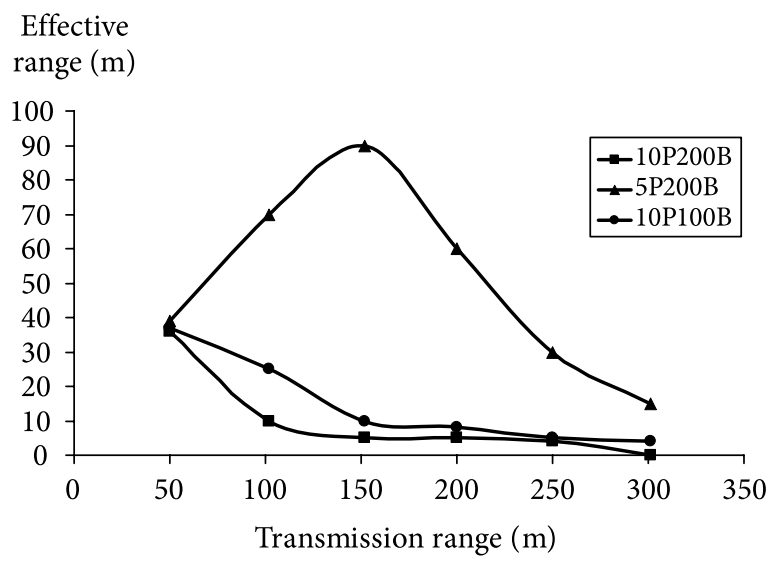

Fig. 3. Effective range of the beacon dissemination protocol 
is relatively close to each other. However, such a noticeable difference can be justified in that in the 5P200B scenario vehicles try to acquire the channel less frequently. It should be stressed that in IEEE 802.11 MAC channel acquisition is the bottleneck of the network's performance. Disseminating fewer messages (beacons), even though the messages are larger, reduces the number of probable collisions, and hence the effective range increases.

The aforementioned observation suggests that if each vehicle aggregates the status of the neighbours in its beacons and instead disseminates beacons less frequently, the effective range of the disseminating protocol improves considerably. On the other side, it leads to a better safety provisioning: even though a vehicle may not receive a beacon from a sender itself directly, it may receive the status through beacons coming from other vehicles. This idea may be invoked when the network becomes very saturated in some situations like a dense road traffic as well as when the VANET's data traffic increases unexpectedly. Instead, the transmission interval of the beacon dissemination protocol can be increased.

\subsection{The Beaconing Rate of the Beacon Dissemination Protocol}

In the second step, we show how well the beacon dissemination protocol behaves in terms of message delivery. Recall that because of single-hop dissemination policy delays are turned out to be sufficient. Also, as previous works found out (Torrent-Moreno et al. 2004; Yousefi et al. 2007a), delivery rates decrease dramatically when the distance form the sender increases. This is mainly due to the CSMA inherent of the MAC layer and to the absence of ACK mechanisms (CTS/RTS) in the broadcast mode of IEEE 802.11. In addition, fairness issue is of great importance which is not evaluated in the previous works. Lack of fairness may spoil the efficacy of dissemination protocols even though they offer very high delivery rates to some vehicles. Fig. 4 depicts the beaconing rate of the implemented beacon dissemination protocol for each vehicle (numbered from 0 to 559) in different scenarios.

As it is followed from the figure, the beacon dissemination protocol is unable to provide adequate fairness to all vehicles. Surprisingly, the vehicles located in the middle of the vehicle platoon are offered the poorest beaconing rates while logically they should be offered the best beaconing rate because they have more neighbours and, thus, are more at risk. Figure presents 8 low peaks, each one representing the vehicle positioned in the middle of each lane. This can be explained by the fact that vehicles in the middle of each lane suffer from heavier contention because of more neighbours they have. By changing the scenario resulting in a lower beacon message size (in Fig. 4 b) or a lower beacon transmission interval (in Fig. $4 \mathrm{c}$ ), the beaconing rate tends to be smoother.

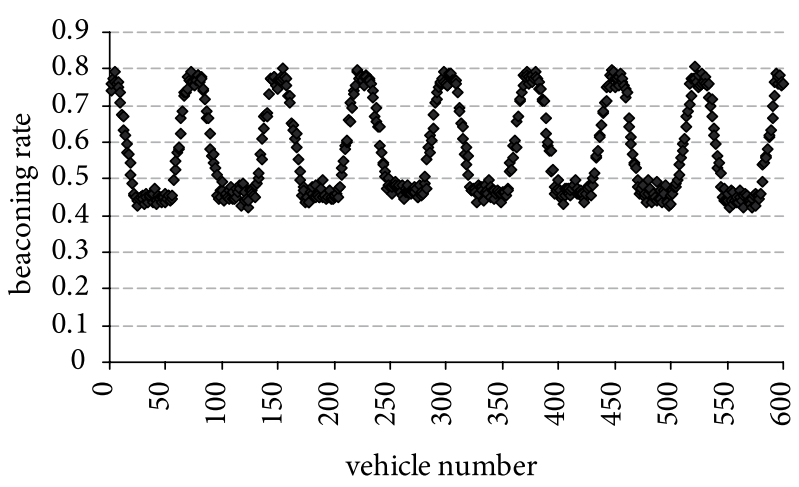

(a) $10 \mathrm{P} 200 \mathrm{~B}$

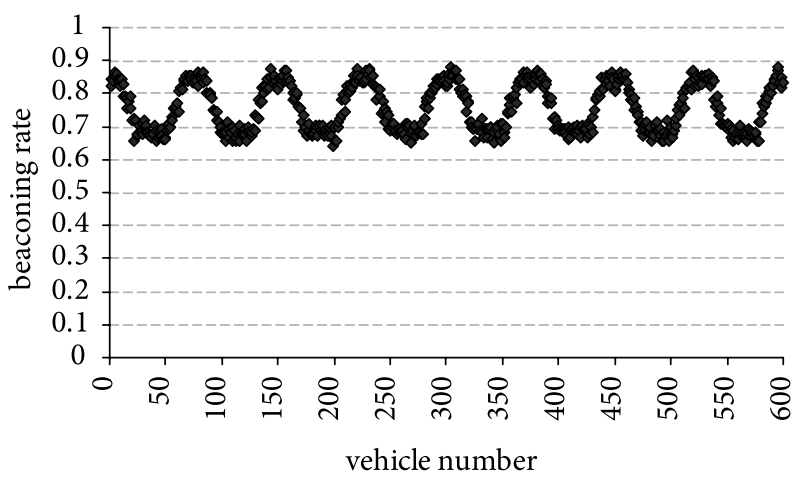

(b) $10 \mathrm{P} 100 \mathrm{~B}$

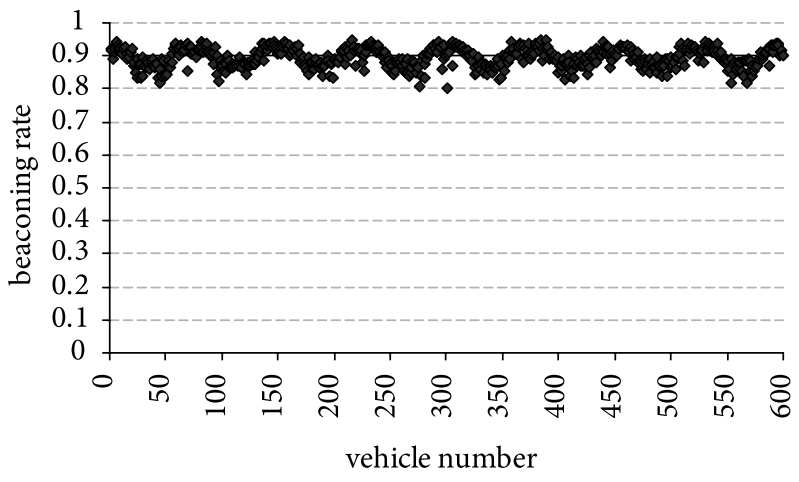

(c) 5 P200B

Fig. 4. Beaconing rate of vehicles in different scenarios (transmission range $=150 \mathrm{~m}$ )

Furthermore, Fig. 5 shows that for a fixed scenario, as the transmission range of the protocol decreases, the beaconing rate becomes fairer. But it comes at the expense of lower effective range which may be risky in safety side. Fig. 4 and Fig. 5 show that each point in the curves associates with an individual vehicle.

\subsection{The Effective Range of Safety Applications}

Chapter 2 showed that the performance of beacon based safety applications might be different from that of the beacon dissemination protocols. In this section, we intend to find the effective range for typical safety applications introduced in Chapter 2. Toward this aim, using the given QoS satisfaction levels (i.e. $\alpha$ ), we find the desired threshold for $P_{c o m}$ from Equation (1) and then measure the effective range in our simulation using delivery rate data in each 


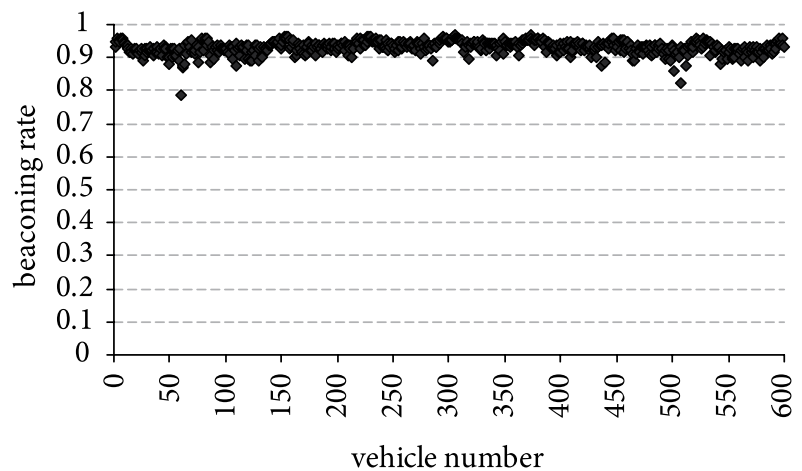

Transmission range $=50 \mathrm{~m}$

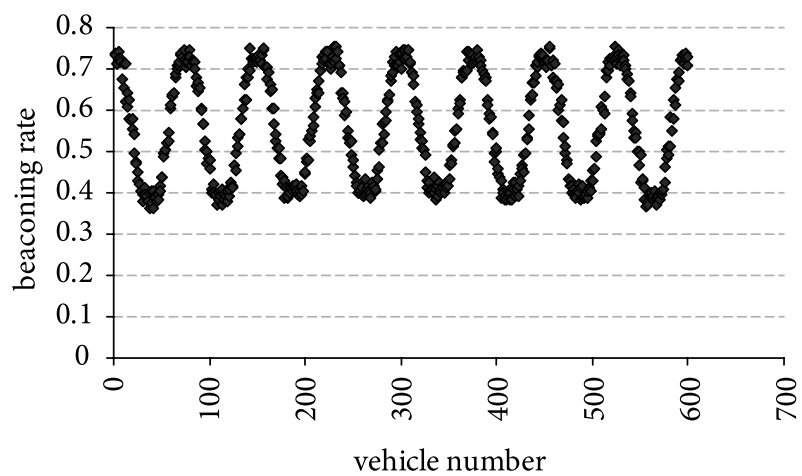

Transmission range $=250 \mathrm{~m}$

Fig. 5. Beaconing rate of vehicles in different transmission ranges (the 5P200B scenario)

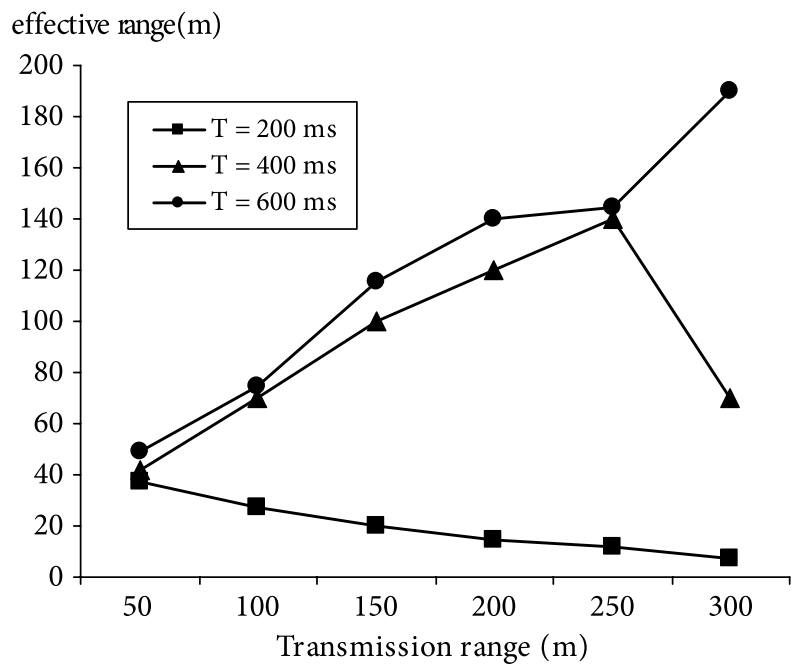

(a) $\alpha=95 \%$

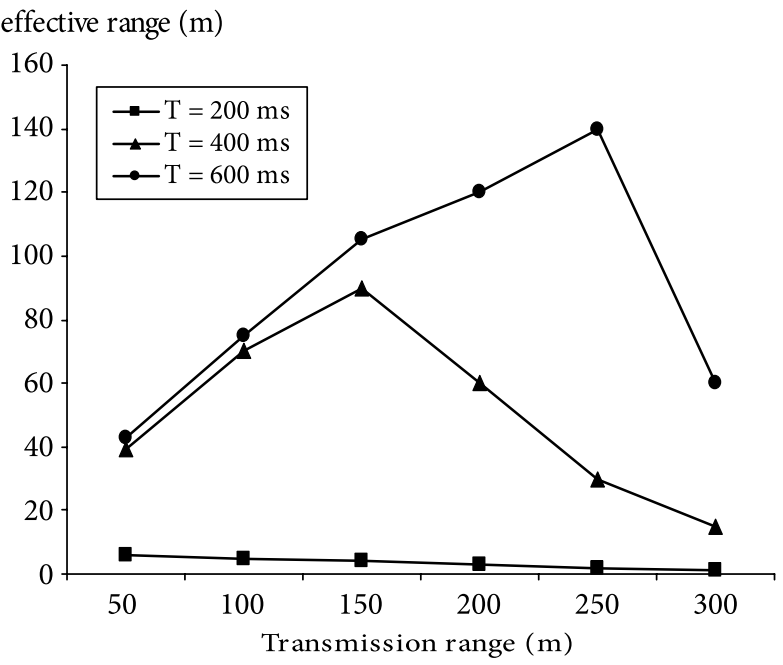

(b) $\alpha=99 \%$

Fig. 6. Application's effective range versus vehicle's transmission range for different values of $\alpha$ and $T$ in the 5P200B scenario

scenario. As a selected result, for different values of $\alpha$ and T, Fig. 6 (a, b) shows the effective ranges versus vehicles' transmission range for the 5P200B scenario. The figure suggests that driver-assistant safety applications (which demand $T=400 \mathrm{~ms}, 600 \mathrm{~ms}$ ) can be deployed with effective ranges from 100 to 200 meters. These effective ranges can be considered satisfactory for many applications.

However, when $T=t=200 \mathrm{~ms}$, the effective ranges are very low and hardly become larger than the average intervehicle distance. As a result, automatic safety applications can not be deployed at least with the MAC layers utilized in our simulation. Fig. 6 further shows that by increasing vehicle's transmission range, there is an increasing trend in application's effective range until the delivery rate of its corresponding beacon dissemination protocol (normally implemented in the MAC layer) falls below the required $P_{\text {com }}$, computed from Equation (1). As this desired threshold is higher, i.e. for smaller values of $T$, the decreasing trend starts in lower transmission ranges, which is expected. Beyond that threshold, increasing vehicle's transmission range leads to decreasing application's effective range.

\section{Conclusions}

1. To facilitate an accurate performance evaluation of beacon based safety applications in VANETs, we introduced two new metrics called beaconing rate and effective range. These metrics take into account distinctive characteristics of safety scenarios such as fairness and coverage.

2. We study the relationship between the performance of beacon dissemination protocols and that of safety applications.

3. Disseminating aggregated status information about neighbours by a vehicle leads to higher performance in both safety and networking aspects. Moreover, driver-assistant safety applications show promises to be implemented in near-term.

\section{References}

Adler, C. J. 2006. Information dissemination in vehicular ad hoc networks. Master's thesis, Univ. of Munich, Germany.

Bai, F.; Krishnan, H. 2006. Reliability analysis of DSRC wireless communication for vehicle safety applications, in IEEE 
Proceedings of Intelligent Transportation Systems Conference (ITSC 2006, IEEE), Toronto, Canada, 355-362.

DOT HS 809 767. 2003. Traffic Safety Facts 2003. 12 p. Available from Internet: <http://www-nrd.nhtsa.dot.gov/Pubs/ 809767.pdf>.

DOT HS 809 859. 2005. Vehicle Safety Communications Project Task 3 Final Report: Identify Intelligent Vehicle Safety Applications Enabled by DSRC. 156 p. Available from Internet: $<$ http://www-nrd.nhtsa.dot.gov/pdf/nrd-12/1665CAMP3web/images/CAMP3scr.pdf $>$.

FCC 03-324: 2004. Federal Communications Commission Report and Order. 78 p. Available from Internet: $<$ http://fjallfoss.fcc. gov/edocs_public/attachmatch/FCC-03-324A1.pdf >.

Füßler, H.; Torrent-Moreno, M.; Transier, M.; Festag, A.; Hartenstein, H. 2005. Thoughts on a protocol architecture for vehicular ad-hoc networks, in Proceedings of the 2 nd International Workshop on Intelligent Transportation (WIT), Hamburg, Germany, 41-45.

GloMoSim Network Simulator. 2008. Available from Internet: $<$ http://pcl.cs.ucla.edu/projects/glomosim $>$.

IEEE Standard 802.11p. 2004. Wireless Access in Vehicular Environments.

Integrated Project PReVENT. 2008. Available from Internet: $<$ http://www.prevent-ip.org $>$.

InternetITS Consortium Project. 2008. Available from Internet: $<$ http://www.internetits.org $>$.

Jarašūnienè, A. 2007. Research into intelligent transport systems (ITS) technologies and efficiency, Transport 22(2): 61-67.

Korkmaz, G.; Ekici, E.; Özgüner, F; Özgüner, Ü. 2004. Urban multi-hop broadcast protocol for inter-vehicle communication systems, in VANET'04: Proceedings of the 1st ACM International Workshop on Vehicular Ad hoc Network, Philadelphia, PA, USA, 76-85.

Networks on Wheels (NOW) Project. 2008. Available from Internet: <http://www.network-on-wheels.de>.

Resta, G.; Santi, P.; Simon, J. 2007. Analysis of multi-hop emergency message propagation in vehicular ad hoc networks, in $\mathrm{Mo}$ biHoc'07: Proceedings of the 8th ACM International Symposium on Mobile Ad hoc Networking and Computing, 140-149.

Roess, R. P.; Prassas, E. S.; McShane, W. R. 2004. Traffic Engineering, 3rd edition, Pearson Prentice Hall, Upper Saddle River, New Jersey. 786 p.

Torrent-Moreno, M.; Jiang, D.; Hartenstein, H. 2004. Broadcast reception rates and effects of priority access in 802.11based vehicular ad-hoc networks, in VANET'04: Proceedings of the 1st ACM International Workshop on Vehicular Ad hoc Network, Philadelphia, PA, USA, 10-18.

Torrent-Moreno, M.; Santi, P.; Hartenstein, H. 2005. Fair sharing of bandwidth in VANETs, in VANET'05: Proceedings of the 2nd ACM International Workshop on Vehicular Ad hoc Networks, 49-58.

Xu, Q.; Mak, T.; Ko, J.; Sengupta, R. 2004. Vehicle-to vehicle safety messaging in DSRC, in VANET'04: Proceedings of the 1st ACM International Workshop on Vehicular Ad hoc Network, Philadelphia, PA, USA, 19-28.

Yin, J.; ElBatt, T.; Yeung, G.; Ryu, B.; Habermas, S.; Krishnan, H.; Talty, T. 2004. Performance evaluation of safety applications over DSRC vehicular ad hoc networks, in VANET '04: Pro- ceedings of the 1st ACM International Workshop on Vehicular Ad hoc Network, Philadelphia, PA, USA,1-9.

Yousefi, S.; Altman, E.; El-Azouzi, R.; Fathy, M. 2008. Improving connectivity in vehicular ad hoc networks: an analytical study, Computer Communication 31(9): 1653-1659.

Yousefi, S.; Bastani, S.; Fathy, M. 2007a. On the performance of safety message dissemination in vehicular ad hoc networks, in IEEE: Proceedings of 4th European Conference on Universal Multiservice Networks ECUMN'2007, Toulouse, France, 377-390.

Yousefi, S.; Fathy, M.; Benslimane, A. 2007b. Performance of beacon message dissemination protocols in vehicular ad hoc networks (VANETs), Journal of Zhejiang University Science A 8(12): 1990-2004.

Yousefi, S.; Mousavi, M. S.; Fathy, M. 2006. Vehicular ad hoc networks (VANETs): challenges and perspectives, in IEEE: Proceedings of 6th International Conference on ITS Telecommunications (ITST2006), Chengdu, China, 761-766. 